\title{
Pulmonary hypertension following L-lysine ibuprofen therapy in a preterm infant with patent ductus arteriosus
}

\author{
Carlo Bellini, Francesco Campone, Giovanni Serra
}

$\infty$

See related article page $\mathrm{I} 855$

\section{ABSTRACT}

Patent ductus arteriosus is one of the most common congenital abnormalities found in premature infants. Ibuprofen, a nonsteroidal drug that is commonly used as an antipyretic, analgesic and anti-inflammatory agent, is also used to induce closure of symptomatic patent ductus arteriosus in preterm infants. Recently, we gave L-lysine ibuprofen to a preterm infant with respiratory distress to induce closure of a patent ductus arteriosus, and the infant experienced pulmonary hypertension. Only 3 cases of pulmonary hypertension following early administration of an ibuprofen solution buffered with tromethamine have previously been reported. However, this severe side effect has never been observed in multicentre, randomized, double-blind controlled trials, nor in recent reviews or meta-analyses of L-lysine ibuprofen use.

CMAJ 2006;174(13):1843-4

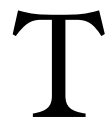

he ductus arteriosus usually closes spontaneously in healthy, full-term infants within 3 days of birth. In preterm infants, it frequently fails to close. Patent ductus arteriosus (PDA) continues to be one of the most common congenital abnormalities found in premature infants. Delayed ductal closure is inversely related to gestational age at birth, and its incidence varies from $20 \%$ in premature infants older than 32 weeks' gestational age up to $60 \%$ in those less than 28 weeks' gestational age. ${ }^{1,2}$ This seems to be related to the incidence of surfactant deficiency. Premature infants who do not present with respiratory distress syndrome seem to achieve PDA closure within a time frame similar to that of term infants. ${ }^{1}$ Premature infants with respiratory distress syndrome experience PDA closure after resolution of respiratory signs and related sequelae. ${ }^{1}$ Ibuprofen, a nonsteroidal drug commonly used as an antipyretic, analgesic and anti-inflammatory agent, has also been used to induce closure of symptomatic PDA in preterm infants. Prostaglandins are synthesized from arachidonic acid by cyclooxygenase. Therefore, NSAIDs that inhibit cyclooxygenase are likely to reduce prostaglandin levels and promote closure of the PDA. Ibuprofen has been shown to be as effective as indomethacin in closing the PDA of preterm infants, but with less renal toxicity. ${ }^{1-6}$
We recently observed a severe adverse effect caused by ibuprofen. Pulmonary hypertension occurred in a preterm infant with respiratory distress syndrome who was given the drug for PDA (Fig. I). Our patient, who was 32 weeks' gestational age and whose birth weight was $1600 \mathrm{~g}$, was I of $\mathrm{I} 69$ preterm infants (age range 24-33 weeks' gestation, birth weight range $450-2365 \mathrm{~g}$ ) who received mechanical ventilation and ibuprofen for PDA closure in our neonatal intensive care unit between January 1997 and June 2005. The patient's mother had not been given glucocorticoids before delivery, nor had she been given ASA or indomethacin. No prolonged premature rupture of the membrane occurred. The patient experienced respiratory distress syndrome, which was treated with surfactant $(200 \mathrm{mg} / \mathrm{kg}$ body weight in 2 separate administrations) and mechanical ventilation (starting parameters: assist/control ventilation, ventilatory rate 60 breaths $/ \mathrm{min}$,

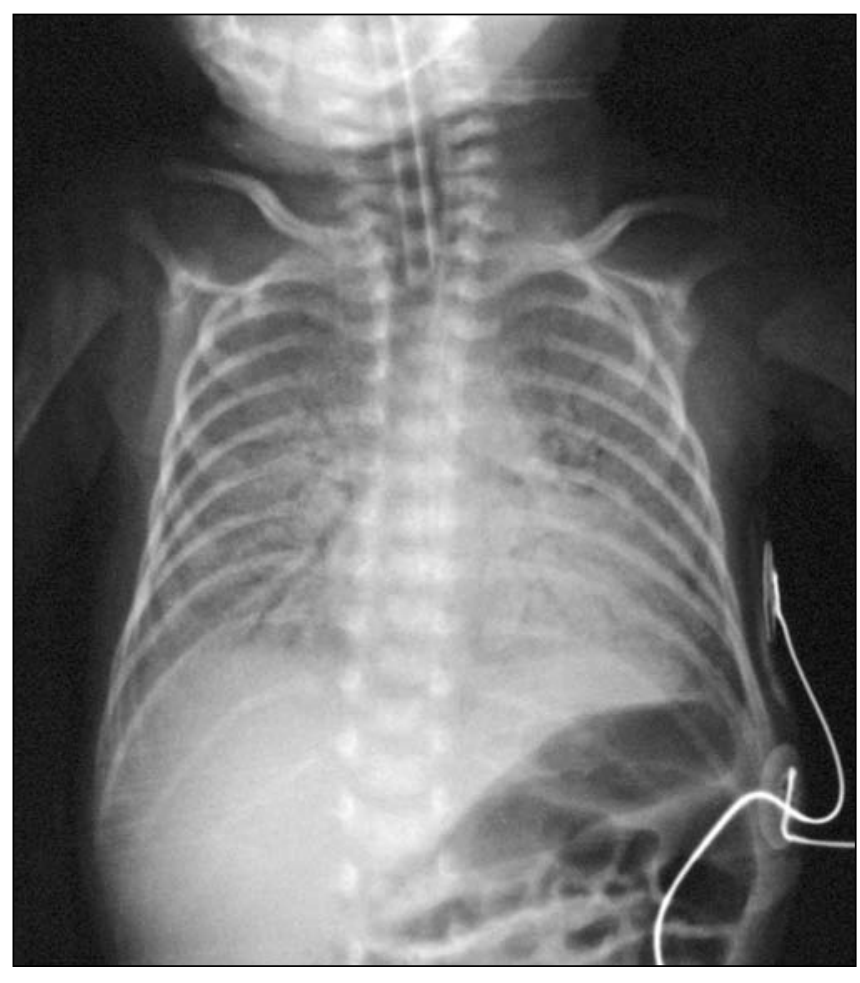

Fig. 1: Anteroposterior chest radiography taken 2 hours after birth. 
peak inspiratory pressure $26 \mathrm{~cm} \mathrm{H}_{2} \mathrm{O}$, positive end-expiratory pressure $5 \mathrm{~cm} \mathrm{H}_{2} \mathrm{O}$, fraction of inspired oxygen $\left[\mathrm{FiO}_{2}\right] \mathrm{I}$ ). Llysine ibuprofen (Io $\mathrm{mg} / \mathrm{kg}$ body weight first dose, then 2 doses of $5 \mathrm{mg} / \mathrm{kg}$ after 24 hours and 48 hours respectively) was administered starting at 72 hours of life under stable respiratory conditions $\left(\mathrm{FiO}_{2} 0.60\right.$ and oxygen saturation $\left.\geq 95 \%\right)$, after echocardiography highlighted hemodynamically significant PDA with a continuous left-to-right shunt but no pulmonary hypertension, and after significant renal impairment, intraventricular hemorrhage, low platelet count and coagulopathy were ruled out. We also ruled out critical pulmonary stenosis, a hypoplastic left ventricle, severe coarctation and transposition of the great arteries.

Within an hour of the second administration of L-lysine ibuprofen, severe hypoxemia was observed with upper-limb oxygen saturation below $70 \%$ during ventilation and $\mathrm{FiO}_{2}$ of I. At the same time, echocardiography showed closure of the ductus arteriosus and a significant right-to-left shunt through the foramen ovale with low right-ventricular output, tricuspid regurgitation and an estimated peak pulmonary arterial pressure of about $50 \mathrm{~mm} \mathrm{Hg}$. These findings clearly indicated the presence of pulmonary hypertension. Inhaled nitric oxide therapy (up to $15 \mathrm{ppm}$ ) was started immediately and led to resolution of the hypoxemia within about 20 minutes, while the $\mathrm{FiO}_{2}$ dropped from I to $0.35-0.40$ within about 50 minutes. Eight hours after nitric oxide was started, echocardiography confirmed the closure of the ductus arteriosus and demonstrated the resolution of pulmonary hypertension. Ibuprofen was discontinued after the second dose. On day 5 of life the patient died because of progressive worsening of clinical conditions that we attributed to generalized sepsis. Postmortem findings were aspecific and compatible with immature lungs that had been ventilated since birth. Arterial vessels showed no thickening or intimal-medial alterations.

\section{Comment}

The occurrence of acute hypoxemia due to pulmonary hypertension after ibuprofen therapy and the prompt recovery from pulmonary hypertension after nitric oxide was administered points to a precise cause-effect event. Our observation confirms the only other previously published report of pulmonary hypertension in 3 preterm infants who were given, within the first 6 hours of life, ibuprofen buffered with tromethamine. ${ }^{7}$ It has been hypothesized that pulmonary hypertension in these 3 infants may have been related either to early administration of the drug, which prevented a normal drop in pulmonary vascular resistance, or to acidification of the ibuprofen solution itself, which results in precipitation and secondary microembolism of the lung. ${ }^{2}$ Other than this report, ${ }^{7}$ pulmonary hypertension after ibuprofen administration has never been reported in any multicentre, randomized, double-blind controlled trial, nor in recent reviews or meta-analyses. ${ }^{1-6}$ There are 2 formulations of ibuprofen available for intravenous use: tromethamine ibuprofen and L-lysine ibuprofen. On the basis of the 3 cases $^{7}$ of pulmonary hypertension after tromethamine ibuprofen administration, Thomas and colleagues ${ }^{2}$ concluded that caution is required only in the case of tromethamine ibuprofen administration. However, since we have now observed a case of pulmonary hypertension after Llysine ibuprofen administration, we conclude that the same caution should be applied to both formulations.

We suggest that pulmonary hypertension in preterm infants receiving therapy for PDA with either ibuprofen formulation must be considered a possible, although rare, severe side effect. We also suggest that echocardiographic evaluation should be performed on all preterm infants before ibuprofen therapy is started to rule out pre-existing pulmonary hypertension. Finally, in line with a recent report, ${ }^{3}$ we conclude that further trials addressing potential adverse effects of ibuprofen, including pulmonary hypertension, are needed.

This article has been peer reviewed.

All of the authors are from the Neonatal Intensive Care Unit, Department of Pediatrics, University of Genoa, Gaslini Institute, Genoa, Italy.

Competing interests: None declared.

Contributors: All of the authors contributed equally to the conception, design, conduct, analysis and writing of this paper and have approved the final version for publication.

\section{REFERENCES}

I. Wyllie J. Treatment of patent ductus arteriosus. Semin Neonatol 2003;8:425-32.

2. Thomas RL, Parker GC, Van Overmeire B, et al. A meta-analysis of ibuprofen versus indomethacin for closure of patent ductus arteriosus. Eur J Pediatr 2005; I64: I35-40.

3. Shah SS, Ohlsson A. Ibuprofen for the prevention of patent ductus arteriosus in preterm and/or low birth weight infants [Cochrane Review]. In: The Cochrane Library; Issue 2, 2005. Oxford: Update Software.

4. Gournay V, Roze JC, Kuster A, et al. Prophylactic ibuprofen versus placebo in very premature infants: a randomised, double-blind, placebo-controlled trial. Lancet 2004;364:I939-44.

5. Van Overmeire B, Allegaert K, Casaer A, et al.; Multicentre Ibuprofen Prophylaxis Study (MIPS) Investigators. Prophylactic ibuprofen in premature infants: a multicentre, randomised, double-blind, placebo-controlled trial. Lancet 2004;364:1945-9.

6. Van Overmeire B, Smets K, Lecoutere D, et al. A comparison of ibuprofen and indomethacin for closure of patent ductus arteriosus. N Engl J Med 2000;343:674-8r.

7. Gournay V, Savagner C, Thiriez G, et al. Pulmonary hypertension after ibuprofen prophylaxis in very preterm infants. Lancet 2002;359:I486-8.

Correspondence to: Dr. Carlo Bellini, Dipartimento di Pediatria, Università di Genova, Instituto G. Gaslini, Largo G. Gaslini, 5, I6I47 Genova, Italy; fax +39 Io 3770675;

carlobellini@ospedale-gaslini.ge.it 